\title{
On the extension of orders in ordered modules: Corrigenda
}

\section{P. Ribenboim}

The author has noticed a faulty argument in his paper [1]; it occurs on $p .85$ and requires the following changes:

At the end of line 9 , for $\left(Q_{i}\right)_{i=1,2, \ldots}$ read $\left(Q_{i}\right)_{i \in I}$;

in line 10, the equation should read $Q=\underset{i \in I}{U} Q_{i}$;

lines 15 to 20 should be replaced by the following:

then there exists some $n$-tuple $\delta=\left(\delta_{1}, \ldots, \delta_{n}\right)$ such that for every $i_{0}$ there exists $i \geq i_{0}$ with $\varepsilon^{i}=\delta$. Then $\delta_{1} a_{1}, \ldots, \delta_{n} a_{n}$ are positively independent over $Q$; for if $r_{j} \in P_{R}$ and $\sum_{j=1}^{n} r_{j} \delta_{j} a_{j} \in Q$ then there exists $i_{0}$ such that $\sum_{j=1}^{n} r_{j} \delta_{j} a_{j} \in Q_{i_{0}}$, and for appropriate $i \geq i_{0}$ it follows that $r_{j}=0$ for $j=1, \ldots, n$.

\section{Reference}

[1] P. Ribenboim, "On the extension of orders in ordered modules", BuZZ. Austral. Math. Soc. 2 (1970), 81-88.

Queen's University,

Kingston, Ontario.

Received 10 November 1970. 